University of Nebraska - Lincoln

DigitalCommons@University of Nebraska - Lincoln

Faculty Publications from the Department of Electrical \& Computer Engineering, Department Electrical and Computer Engineering

2002

\title{
A Multiwavelength Airborne Polarimetric Lidar for Vegetation Remote Sensing: Instrumentation and Preliminary Test Results
}

Songxin Ran

University of Nebraska-Lincoln

Ram M. Narayanan

University of Nebraska-Lincoln

Follow this and additional works at: https://digitalcommons.unl.edu/electricalengineeringfacpub

Part of the Electrical and Computer Engineering Commons

Ran, Songxin and Narayanan, Ram M., "A Multiwavelength Airborne Polarimetric Lidar for Vegetation Remote Sensing: Instrumentation and Preliminary Test Results" (2002). Faculty Publications from the Department of Electrical and Computer Engineering. 156.

https://digitalcommons.unl.edu/electricalengineeringfacpub/156

This Article is brought to you for free and open access by the Electrical \& Computer Engineering, Department of at DigitalCommons@University of Nebraska - Lincoln. It has been accepted for inclusion in Faculty Publications from the Department of Electrical and Computer Engineering by an authorized administrator of DigitalCommons@University of Nebraska - Lincoln. 


\title{
A Multiwavelength Airborne Polarimetric Lidar for Vegetation Remote Sensing: Instrumentation and Preliminary Test Results
}

\author{
Songxin Tan and Ram M. Narayanan \\ Department of Electrical Engineering, University of Nebraska-Lincoln \\ 209 N Walter Scott Engineering Center, Lincoln, NE 68588-0511, USA \\ Tel: (402)472-5141, FAX: (402)472-4732, Email: ram@doppler.unl.edu
}

\begin{abstract}
Several spaceborne and airborne lidar systems have been launched for vegetation canopy studies. Previous research has shown that lidars are useful tools for remote sensing of vegetation architecture. To support its Airborne Remote Sensing Program, the University of Nebraska has developed a multiwavelength airborne polarimetric lidar system. This system employs a Nd:YAG laser which emits radiation at two wavelengths: the fundamental at $1064 \mathrm{~nm}$ and the frequency-doubled at $532 \mathrm{~nm}$. Both laser beams are highly linearly polarized (100: 1 extinction ratio) and have a beam divergence angle of $\sim 4 \mathrm{mrad}$. The receiver consists of four channels, which enable dual-wavelength and dual-polarization detection. In addition to the polarimetric information that could be gathered, this lidar system also has ranging capability and is able to record the whole lidar waveform. Thus, our lidar is capable of performing studies of vegetation canopy structure as well as characterization of vegetation depolarization. The system has been packaged to fly aboard a Piper Saratoga aircraft from a height of $1000 \mathrm{~m}$.

In this paper, we will present the details of the lidar system design, instrumentation, the system alignment and preliminary ground test results.
\end{abstract}

\section{INTRODUCTION}

Lasers have long been used in vegetation remote sensing [1]. Previous applications involve the laser altimeters, through which a 3-D structure of vegetation is obtained. This has great advantage over passive optical remote sensing, which do not have the ability to acquire vertical height distributions. With small footprint laser altimeters, a true digital elevation map (DEM) can be obtained by removing the ground vegetation. Previous laser altimeters typically have small footprint, rangeonly detection and work at low altitude. With the recent technology development, a lot of new lidar systems appeared. For example, the Scanning Lidar Imager of Canopy by Echo Recovery (SLICER) is a large footprint, high altitude system that can digitize the entire return laser pulse (lidar waveform). Using large footprint lidar such as SLICER, an integrated canopy structure/substructure could be obtained from the lidar waveform. The proposed Vegetation Canopy Lidar (VCL) is a space borne lidar system. VCL will enable monitoring of vegetation canopy in a global scale. The vegetation canopy structure has great impact on the interaction between vegetation and atmosphere. For example, the heat and mass transportation, transpiration, photosynthesis and other biophysical or biochemical mechanisms are functioned through the vegetation canopy. The research of canopy is also helpful to understand the microclimate generated by vegetation. The Airborne Laser Polarimetric Sensor (ALPS), on the other hand, is a system that tried to investigate the polarization property of vegetation under laser illumination [2]. The polarization property of vegetation is extremely difficulty to obtain under solar illumination. Using active laser illumination, interesting depolarization properties of coniferous and broadleaf trees and good correlation between depolarization and nitrogen concentration were obtained [2]. Unfortunately, the ALPS system did not acquire the entire lidar waveform, and was unable to obtain the 3-D structure of vegetation.

To support its airborne remote sensing program, a Multiwavelength Airborne Polarimetric Lidar (MAPL) has been developed at the University of Nebraska. This system is required to be able to acquire the lidar waveform, and has polarimetric abilities at the same time. The sensor will fly on a Piper Saratoga aircraft, at an altitude of about $1000 \mathrm{~m}$. The primary function of this lidar is to study the vegetation canopy, using both 3-D structure and depolarization information. We also plan to estimate the biomass, leaf area index (LAI), etc; and study the vegetation stress conditions.

\section{SYSTEM DESCRIPTION}

The MAPL system is composed of several sub-systems. They can be approximately divided into three main parts: the laser source, the optical receiver assembly and the data acquisition and processing hardware and software. The block diagram is shown in Fig. 1.

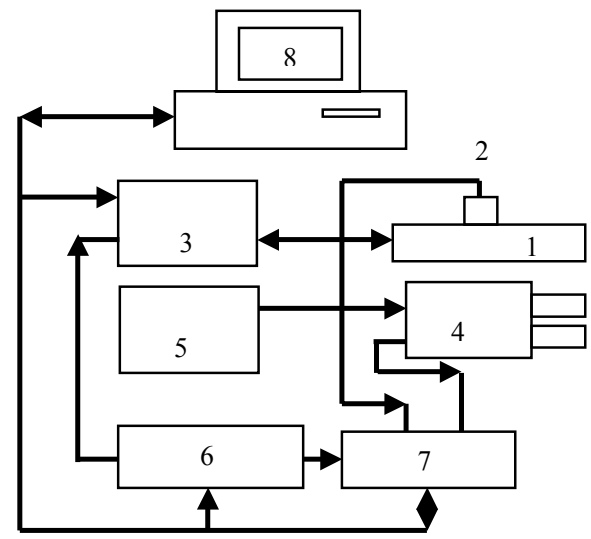

Figure 1. Block diagram of MAPL. 1. Laser head, 2. Si detector, 3. Laser power supply and control, 4 . optical receiver package, 5 . high voltage supply for PMT detector, 6. Digital delay pulse generator, 7. Digitizer and 8. computer.

A computer controls a digital delay/pulse generator through a RS-232 interface. TTL signals are then generated by the 
delay generator. One TTL signal is sent to trigger the Qswitch of the laser; another TTL signal is delayed and sent to trigger the digitizer. The delay time is determined by the range of the target. The laser sends out two laser pulses at $1064 \mathrm{~nm}$ and $532 \mathrm{~nm}$ simultaneously. On top of the laser head, there is a $\mathrm{Si}$ detector to monitor the laser output. The laser pulses are reflected by the vegetation and/or the ground, and received by four PMT detectors. These detectors enable detection at both copolarized and cross-polarized direction at the two wavelengths. Outputs from the PMTs are A/D converted by a fast digitizer and then sent to the computer through a PCI interface card. The ranging ability is achieved by precisely timing the transmission and receiving of the laser pulse. Data are stored in a hard drive and post-processed by the computer to get information of range and vegetation height and 3-D structure.

\section{A. Laser}

The MAPL employs a Bigsky CFR-400 Nd:YAG laser. This is a multi-wavelength pulsed laser system, and is designed to be compact, rigid and stable, and suitable for small aircraft applications. This laser has two outputs: one at $1064 \mathrm{~nm}$ and one at $532 \mathrm{~nm}$, emitting from two separate apertures. Special optical wedge assembly is provided inside the laser module to make sure that the two beams are parallel. The two beams are linearly polarized in the horizontal direction. The polarization purities are $99 \%$ for both wavelengths. The beams have divergence angle of about 4 mrad, which will form a laser footprint of about $4 \mathrm{~m}$ at an altitude of $1000 \mathrm{~m}$. The highest pulse repetition rate is $10 \mathrm{~Hz}$, and the maximum output energy for each wavelength is 100 $\mathrm{mJ} /$ pulse. The near field beam diameter is about $6 \mathrm{~mm}$ for each wavelength. The pulse width is $10 \mathrm{~ns}$ which gives a best range resolution of $1.5 \mathrm{~m}$. To ensure accurate timing, trigger of flash lamp and Q-switch are separately controllable.

A Si PIN detector from Thorlabs is O-ring sealed and attached externally on top of the laser head, which will monitor the relative output laser energy at 1064-nm. This is used as a reference to normalize the received waveform.

The power supply unit and cooling group unit are integrated into a Mini-ICE (integrated cooler and electronics) subsystem. It is a compact and rugged sub-system designed to operate in hard outdoor environment. A remote control box is provided to run all the controls on the laser. A RS-232 interface is also provided for computer controls.

\section{B. Receiving Optics}

Totally four receiving channels are employed in the MAPL, which enable dual-wavelength and dual-polarization detection. There are two channels, one copolarized and one crosspolarized, for the $1064 \mathrm{~nm}$ light; and the same for $532 \mathrm{~nm}$ light. Adding more receiving channels will enable the measurement of Stokes parameters, which will fully describe the polarization state. However, the circularly polarized light from vegetation is very weak, under linear polarized laser illumination. Useful information will be extremely difficult to retrieve because of the low signal to noise ratio (SNR). Thus such ability is not integrated in the system [3].

The four receiving apertures are $25 \mathrm{~mm}$. There is a polarizer, an optical interference filter and a single planoconvex lens with a $65 \mathrm{~mm}$ focal length, inside each receiver. The receiving light is diffused onto the PMT detection plane, instead of imaging onto it. Here we adopted the same structure as used in the ALPS system [2]. The advantage of the socalled straight through structure is to minimize the reflection and refraction optics inside the receivers, thus decrease possible modifications to the polarization state of the backscattered light. The 1064-nm polarizers inside the receivers are from Corning Polarcor and the 532-nm polarizers are from Polaroid, both of them have polarization extinction ratios higher than 1,000:1. We used narrow band interference filters from Andover. The filters have FWHM bandwidths of 1 $\mathrm{nm}$. The narrow band filters will greatly reduce the background noise. The peak transmission for 1064-nm filter is $\sim 38 \%$, and for $532-\mathrm{nm}$ filter is $\sim 47 \%$. Each detector has a field stop at the focal plane of the lens. The field stops define the detector FOVs to be $6 \mathrm{mrad}$, slightly larger than the laser beam divergence angle.

High sensitivity and ultra-fast PMTs from Hamamatsu are used as photodetectors. R632-01 is used for $1064 \mathrm{~nm}$ light detection, and R1464 is used for $532 \mathrm{~nm}$ light, making good use of their spectrum response.

\section{Data Acquisition and Processing}

We used a model 555 digital delay/pulse generator from Berkeley Nucleonics as a timing source. It is used to generate the control TTL signals to trigger the laser and the digitizer. The timing accuracy of the delay generator is $1 \mathrm{~ns}$. For A/D conversion, we chose two 8-bit digitizing cards, DC256 and DC110, from Acqiris. DC256 has four input channels and a sampling rate of $500 \mathrm{MS} / \mathrm{s}$. It is used to digitizing the PMTs' outputs. DC 110 has one channel and samples upto $1 \mathrm{GS} / \mathrm{s}$. It is used for reference signal digitizing. Both cards are housed inside a modular CompactPCI crate, and connected to the computer through a PCI interface card with $100 \mathrm{Mb} / \mathrm{s}$ transfer rate. The Acqiris digitizers employ an internal time trigger interpolator with 5 ps resolution, which is used to assist timimg calibration and trigger positioning. The accurate timing ability of digitizer will improve the range measurement accuracy, and help obtain accurate lidar waveforms, which contain vegetation canopy vertical structures.

Labview program is used to control the whole system. Functions of the program include control of the digital delay generator, the laser, the digitizer, data recording and real time waveform display. Data are recorded and post processed.

\section{SYSTEM ALIGNMENT}

System alignment is difficult because there are totally six optical axes, four receivers and two laser beams. They must be in perfect alignment. The two laser beams were already made collinear in the factory. The task remained to align the four detectors, and make sure the detectors and the laser beams are parallel. Another task was to align the polarization direction of 
the polarizers, making sure that they were at the copolarized and cross-polarized direction as expected.

For alignment purpose, a special halogen lamp assembly, MR6 from Gilway, was employed. This lamp assembly had a polished aluminum reflector to increase forward irradiance. To align the four detectors, the system was brought into a dark room. The lamp assembly was placed inside the receiver, back at the detector plane. Lamplight passed through the receiving optics and illuminated a white board 10 meters in front of the receivers. The circular light spot on the board was the image of the field stop. The position of the spot was carefully marked and the center was estimated. Because the four receivers were $70 \mathrm{~mm}$ away from each other, so the centers of the four spots should also be $70 \mathrm{~mm}$ apart from each other, if they are to be parallel. The receiver barrels were carefully adjusted to make sure that the four spot centers are $70 \mathrm{~mm}$ away from each other. Thus these receivers were in alignment. Using the lamp assembly, we were able to accurately define the detector FOVs. In our case, the board was at $10 \mathrm{~m}$ distance, the light spot had a diameter of $60 \mathrm{~mm}$. So the FOV was $6 \mathrm{mrad}$. Changing the diameter of the field stops will change the FOVs of the system.

The lamp assembly also facilitated the polarization direction alignment. The lamp was put at the detector plane and another polarizer with horizontal polarization direction was placed in front of the detector. The optical axis of the polarizer inside the detector was adjusted until maximum light intensity was observed through the second polarizer. Thus we obtain the co-polarized channel. Cross-polarized channel was obtained when minimum light intensity was observed. To observe the $1064 \mathrm{~nm}$ light, we used an infrared viewer.

Alignment of laser beams and receivers was performed in the field. At that time, three PMTs were already installed. One green channel was left and the lamp assembly was still inside it. A $6 \mathrm{~m} \times 6 \mathrm{~m}$ target was setup $1000 \mathrm{~m}$ away from the lidar. At the target site, a telescope was used to observe the lamplight from the lidar. The position of the spot was marked on the target. Then we turn on the laser and let the laser shoot at the mark on the target. The signal from the completed three channels were displayed and recorded. Then slightly adjust the laser position until maximum outputs were obtained from the completed channels. Thus the laser beams and the detector are in perfect alignment.

\section{TEST RESULTS AND CONCLUSIONS}

After alignment, the lidar system was tested on the ground. The test site was chosen in Mead, Nebraska. The test scene is shown in Fig. 2. There were five deciduous trees at a distance $1010 \mathrm{~m}$ away from the lidar, and some coniferous bushes and a small hill with dry grass at about $1080 \mathrm{~m}$. The area that the lidar scanned over is marked by a rectangular in Fig. 2. Lidar scanned from the left to the right, with 17 scan lines inside the area. Fig. 3 shows the range intensity image of these scan lines, where (a) is the $1064 \mathrm{~nm}$ copolarized return, (b) is the $1064 \mathrm{~nm}$ cross-polarized return, (c) is the $532 \mathrm{~nm}$ copolarized return and (d) is $532 \mathrm{~nm}$ cross-polarized return. The returns at about $1010 \mathrm{~m}$ are from these tree trunks and returns at about $1080 \mathrm{~m}$ are from bushes and dry grasses. Fig. 3 (a), (b) and (c), (d) compose the so-called orthogonal state contrast image in polarimetric imaging. At present time, these lidar returns are not polarimetrical calibrated. Polarimetric calibration using canvas tarp is still underway.
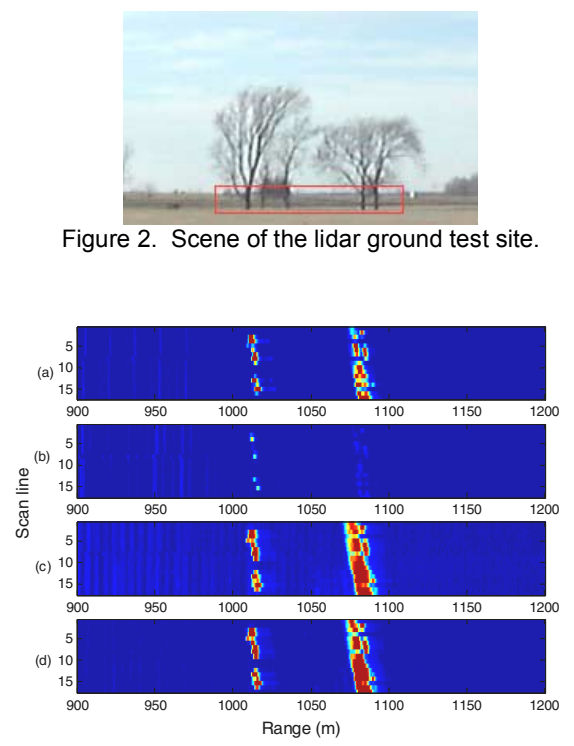

Figure 3. Lidar return from the above scene.

Preliminary ground test results shows that our lidar system functions quite well. At a range of more than $1000 \mathrm{~m}$, good SNR are still achieved at both copolarized and cross-polarized channels. Study of the lidar waveform proves that this lidar system can actually resolve objects $1.5 \mathrm{~m}$ away from each other. Polarimetric calibration of the lidar is still underway. After polarimetric calibration, accurate depolarization data will be obtained and used to explain the lidar returns. Polarimetric diversity will enhance detection and assist classification. The MAPL system will be especially useful for applications where targets spectral responses are similar, but the polarization characteristics are different.

\section{ACKNOWLEDGMENT}

The authors would like to thank Dr. James E. Kalshoven of NASA GSFC for his many helps.

\section{REFERENCES:}

[1] J. B. Blair, D. L. Rabine and M. A. Hofton, "The laser vegetation imaging sensor: a medium-altitude, digitizationonly, airborne laser altimeter for mapping vegetation and topography”, J. Photogrammetry and Remote Sensing, vol. 54, pp. 115-121, 1999.

[2] J. E. Kalshoven, P. W. Dabney, "Remote sensing of the earth's surface with an airborne Polarized laser", IEEE Trans. Geoscience and remote sensing, vol. 31, pp. 438-446, 1993.

[3] S. Tan, R. M. Narayanan and J. E. Kolshoven, "Measurement of stokes parameters of materials at 1064-nm and 532-nm wavelengths", Proc. SPIE, vol. 4377, pp. 263$271,2001$. 